\title{
A genomic study of the inter-ORF distances in Saccharomyces cerevisiae
}

\author{
Vicent Pelechano, José García-Martínez and José E. Pérez-Ortín* \\ Departamento de Bioquímica y Biología Molecular, Facultad de Ciencias Biológicas, Universitat de València, CIDr. Moliner 50, E46I00 \\ Burjassot, Spain
}

* Correspondence to: José E. Pérez-Ortín,

Departamento de Bioquímica y Biología Molecular, Facultad de

Ciencias Biológicas, Universitat de València, CIDr. Moliner 50, E46100 Burjassot, Spain.

E-mail: jose.e.perez@uv.es

Received: I March 2006

Accepted: 23 May 2006

\begin{abstract}
The genome of eukaryotic microbes is usually quite compacted. The yeast Saccharomyces cerevisiae is one of the best-known examples. Open reading frames (ORFs) occupy about $75 \%$ of the total DNA sequence. The existence of other, non-protein coding genes and other genetic elements leaves very little space for gene promoters and terminators. We have performed an in silico study of inter-ORF distances that shows that there is a minimum distance between two adjacent ORFs that depends on the relative orientation between them. Our analyses suggest that different kinds of promoters and terminators exist with regard to their length and ability to overlap each other. The experimental testing of some putative exceptions to the minimum length model in tandemly orientated ORF pairs suggests that, in those cases, defects in promoter or terminator functionality exist that provoke transcription of polycistronic mRNAs. Copyright $\odot 2006$ John Wiley \& Sons, Ltd.
\end{abstract}

Keywords: transcription interference; ORF; Saccharomyces cerevisiae; polycistronic; co-regulation

\section{Introduction}

The genome of the yeast Saccharomyces cerevisiae is extremely compact. More than $72 \%$ of the sequence is organized in open reading frames (ORFs). Thus, there is very little space for noncoding signatures, such as promoters, terminators and other elements. The first calculations made by Dujon (1996) showed that, in yeast, ORFs were organized randomly with regard to their respective orientation. About half of the arrangements were tandemly orientated, $25 \%$ convergent and $25 \%$ divergent ORFs. Assuming that genes are nonoverlapping units, the sizes of the promoters and terminators could be calculated from the distances between divergent and convergent ORFs, respectively (Dujon, 1996). This pioneering work determined that the sizes of the average yeast promoter and terminator were of $309 \mathrm{bp}$ and $163 \mathrm{bp}$, respectively. Strictly speaking, these numbers correspond to the sizes of the $5^{\prime}$ and $3^{\prime}$ sequence flanking of the ORFs. This estimation does not make an important error for the terminator, because most of the signals for $3^{\prime}$ mRNA generation are within the transcribed region (Van Helden et al., 2000). However, the identification of a $5^{\prime}$ region of an ORF as a gene promoter is not correct because there is a space between the first nucleotide of the mRNA and the first nucleotide of the ATG: 15-75 bp (Zhang and Dietrich, 2005). Thus, the promoter size would be, on average, about $30 \mathrm{bp}$ shorter (279 bp). These calculations, however, use a very simplistic approach because the distribution of inter-ORF distances is not a Normal (Gaussian) distribution.

Intergenic sequences have, on the other hand, compositional differences with regard to the adjacent ORFs (Dujon, 1996) and with regard to the ORF orientation (Marín et al., 2004), which suggests a functional specialization regarding the ORF orientation. The separation between ORFs and between genes is very important for gene regulation. It is critical that transcription of the adjacent gene does not interfere with the initiation of the 
transcription of the gene immediately downstream (tandem or convergent ORFs) or with the space required for the functionality of two gene promoters (divergent ORFs). It has been shown that transcriptional interference (TI) in natural cases is quite low (Puig et al., 1999) or even absent (Atkins et al., 1994) when two convergent genes coincide in a small region that is, in part, transcribed from both strands. Only when terminators are deleted is a strong TI observed, due to collisions between RNA polymerases (Prescott and Proudfoot, 2002). One reason could be that, in general, the $3^{\prime}$ end formation signals are degenerate, redundant and disperse (Aranda et al., 1998a; Van Helden et al., 2000), which allows for an easy overlap or even the existence of bidirectional signals (discussed in Aranda et al., 1998b). Interestingly, in the case of tandem genes TI seems to be caused by RNA polymerase complexes that initiate transcription from the promoter of the upstream gene and subsequently read through the promoter of the downstream gene. This causes promoter occlusion by disruption of transcription factor binding (Greger et al., 2000; Martens et al., 2004). The potential existence of TI in these cases has apparently guided the evolution of mechanisms to avoid it (Valerius et al., 2002). Strong transcriptional terminator signals, specific factor binding sites and nucleosomal organization have been demonstrated to be required to avoid TI (Greger et al., 2000; Aranda et al., 1998b; Valerius et al., 2002). Closely spaced genes are more prone to TI. These observations prompted us to study the intergenic distances in the entire genome of S. cerevisiae.

We first made a statistical study of the inter-ORF separations for the three types of ORF arrangements. Our conclusion is that a minimum distance exists between two consecutive ORFs and that this minimum and the typical distance for each case are specific for each of the three possible arrangements. This supports Dujon's model of promoter and terminator sizes, although our results provide more accurate estimations. From these analyses, it can be also concluded that there are different subgroups of terminator and promoter sizes for yeast genes. Our specific experimental analysis of the case of tandemly arranged ORFs demonstrates that most of the previously suspected exceptions to the 'minimum distance' rule were false ORFs that are now considered dubious (according to SGD: http://www.yeastgenome.org/) and are likely to be annotation artefacts. Moreover, for the few cases of very short distances, a potential TI on the downstream ORF exists, specifically, mRNAs from the upstream gene invade its promoter and coding regions, producing polycistronic transcripts.

\section{Materials and Methods}

\section{Strains and media}

We used the yeast strains S288c and BQS252 (MATa, ura3-52, derived from FY1679) as laboratory strains, and $\mathrm{T} 73$ as an alternative strain (Querol et al., 1996). Cells were grown in YPD (yeast extract $1 \%$, peptone $2 \%$, glucose $2 \%$ ) in agitation at $28{ }^{\circ} \mathrm{C}$ and recovered by centrifugation at OD 0.5 .

\section{PCR and RT-PCR}

PCR amplifications were performed with genomic DNA that was phenol extracted using Fast-Prep (Bio101, Inc.) and precipitated with ethanol (Hoffman and Winston, 1987). The oligonucleotides were designed to prime from the $3^{\prime}$ region of the first ORF of the tandem to the $5^{\prime}$ region of the second $\mathrm{ORF}$, in order to amplify the intergenic region (see Table 1 for oligonucleotide sequences).

For RT-PCR analyses, RNA samples were also purified by phenol extraction as described (GarcíaMartínez et al., 2004) and, prior to the cDNA synthesis, were treated with DNase I (RNase free, Roche) at $37^{\circ} \mathrm{C}$ for $1 \mathrm{~h}$, phenol-extracted and precipitated with ethanol. The cDNA synthesis was carried with SuperScript II (Invitrogen) for $1 \mathrm{~h}$ at $42{ }^{\circ} \mathrm{C}$ in the presence of RNase OUT (Invitrogen), using an oligo dT as primer. PCR was done using the same oligonucleotides as for DNA.

Both PCR and RT-PCR analysis were done by using Taq DNA polymerase (Biotools) and with the following cycling conditions: $3 \mathrm{~min}$ at $94^{\circ} \mathrm{C}$, 35 cycles of $30 \mathrm{~s}$ at $94^{\circ} \mathrm{C}, 45 \mathrm{~s}$ at $48^{\circ} \mathrm{C}$ and $60 \mathrm{~s}$ at $72^{\circ} \mathrm{C}$ and a single step of $5 \mathrm{~min}$ at $72^{\circ} \mathrm{C}$.

\section{3' RACE}

We used the $3^{\prime}$ RACE method (Frohman et al., 1988) basically with the same conditions for amplification described in RT-PCR. We used the $3^{\prime}$ RACE poly(T) as primer during the cDNA synthesis and $3^{\prime}$ RACE and the gene-specific 
Table I. List of oligonucleotides used in this work

\begin{tabular}{ll}
\hline YPL27IW-D & ATG TCT GCC TGG AGG AAA GC \\
YPL270W-R & GGG TGA CCA AAG ACC GAA AC \\
YHRI30C-D & AAG GAC GCT GAA GAT CAT G \\
YHRI3IC-R & CAT CAA TGA TGC CAA TCG \\
YHRI30C-D2 & GGA GGA CGA CGG TGA CG \\
YMR063W-D & TGC TCA ATG GAA CAG TAC TC \\
YMR064W-R & TAA TGA TCT TTC GAC CGT C \\
YMR064W-DI & TT GCG AGA ATA TAC TGG \\
YMR064W-D2 & AGA ATA CGG GCG CTG TAG G \\
YJL089W-D & AGA GAT AGC AAT TCG GTA G \\
YJL088W-R & GAA GAA AGA AAC GGT GAC \\
YCL008C-D & ATC CAG AAC AGC ACG GAC \\
YCL007C-R & TTG TTC TTG GCC TTA AAC TG \\
YDR462W-D & ACC TTC TAC AAG AAC TCA GC \\
YDR463W-R & AGA ACG CGT ATA TCT TGC \\
YNR057C-D & GGA ACC GAA TGA AGG CAA C \\
YNR056C-R & GAT ACC GAC CCA TGA GCA AC \\
YOR077W-D & GAA GAT GGA ATG CTG CGA AG \\
YOR078W-R & TGC GCT GGC TTG ACT TTC \\
YOR078W-D & TCA AGA CAT GTA ACT TTC G \\
YJRI20W-D & GCG CAA GGA TAT TCC CAT C \\
YJRI2IW-R & ATA GCG GGC AAC TCT GAT TG \\
YDR082W-D & TGA TCT TGA TCC GAA GAA TGG \\
YDR083W-R & GGC TAC TGA TCC TTC GGA AG \\
ACTIID2 & GTA TIT TCA CGC TTA CTG C \\
ACTI-R & TTG GTC TAC CGA CGA TAG ATG \\
Oligo dT & TIT TIT TT TT TT (AGC)(AGCT) \\
3' RACE poly(T) & GGC CAC GCG TCG ACT AG(T) I7 \\
3'RACE & GGC CAC GCG TCG ACT AG \\
\hline & \\
\hline &
\end{tabular}

oligonucleotide (see Table 1) during the PCR amplification.

\section{Northern blot analysis}

We used a $1 \%$ agarose gel with $1 \times$ MOPS and $6.4 \%$ formaldehyde. The samples were transferred to a nylon membrane (Hybond $\mathrm{N}^{+}$, Amersham) by capillarity with $6 \times$ SSC overnight and UVcross-linked. Then the filter was hybridized for $16 \mathrm{~h}$ at $42{ }^{\circ} \mathrm{C}$ in $50 \%$ formamide, $5 \times \mathrm{SSPE}, 5 \%$ dextran sulphate, $0.5 \%$ SDS, $5 \times$ Denhardt and salmon sperm DNA $200 \mu \mathrm{g} / \mathrm{ml}$. We used probes that cover the entire $\mathrm{ORF}$ and were ${ }^{33} \mathrm{P}$ randomprimer labelled with Ready-to-Go (Amersham). The filters were washed twice during $10 \mathrm{~min}$ at $42{ }^{\circ} \mathrm{C}(2 \times$ SSPE and $0.1 \%$ SDS $)$ and once during $15 \mathrm{~min}$ at $65^{\circ} \mathrm{C}(1 \times \mathrm{SSPE}$ and $0.1 \%$ SDS $)$ and exposed to an imaging plate (BAS-MP, Fujifilm).

\section{Statistical procedures}

We calculated the Neperian logarithm of the distance between ORFs (yellow dots in Figure 1). The data were fitted using the non-linear least squares method to the less complex sum of Gaussians that can explain the data properly (red dotted line in Figure 1).

\section{Results}

\section{In silico study of adjacent ORF distances}

We analysed the distance between adjacent ORFs by dividing them into three groups: convergent, divergent and tandemly orientated (Figure 1A). The abrupt slope of the left side of each curve reflects the existence of a minimum distance between ORFs. We noticed that they are distributed, approximately, as a log-Normal curve (dots in Figure 1B-D). Thus, the maximum for each curve represents the most frequent distance observed in each case. This typical distance is 236 , 490 and 402 bp for convergent, divergent and tandem ORFs, respectively. We observed that divergent ORFs are clearly composed of at least two different populations (lines in Figure 1C). Convergent and tandem ORFs form more symmetrical curves, but they can be split up into simpler Gaussian distributions (Figure 1B, D). The two main populations in divergent ORFs are centred at 290 and $771 \mathrm{bp}$. Assuming that the distance represents the space required for two promoters, their sizes would be 145 and 386 bp for the two populations, respectively. For each subpopulation, we calculated the shortest distance as the one that leaves $5 \%$ of the group below it. This value represents an approximation of the minimum separation allowed for two ORFs. These values are shown in Figure 1B-D. A plausible interpretation for such a minimum distance is that gene promoters and terminators behave, at least in part, as solid entities not overlapping with other entities. The length of the promoter and the length of the space in between two promoters would, in any case, also follow a Gaussian distribution when considering the entire yeast genome. Following this reasoning, the minimum length for a gene promoter would be half of the minimum length between divergent ORFs: 79 and $129 \mathrm{bp}$ for the two subpopulations. The minimum length for a gene terminator would be 18 and $37 \mathrm{bp}$ for the two subpopulations of convergent ORFs. In the case of tandem ORFs, the separation would be a composition of a promoter plus a 

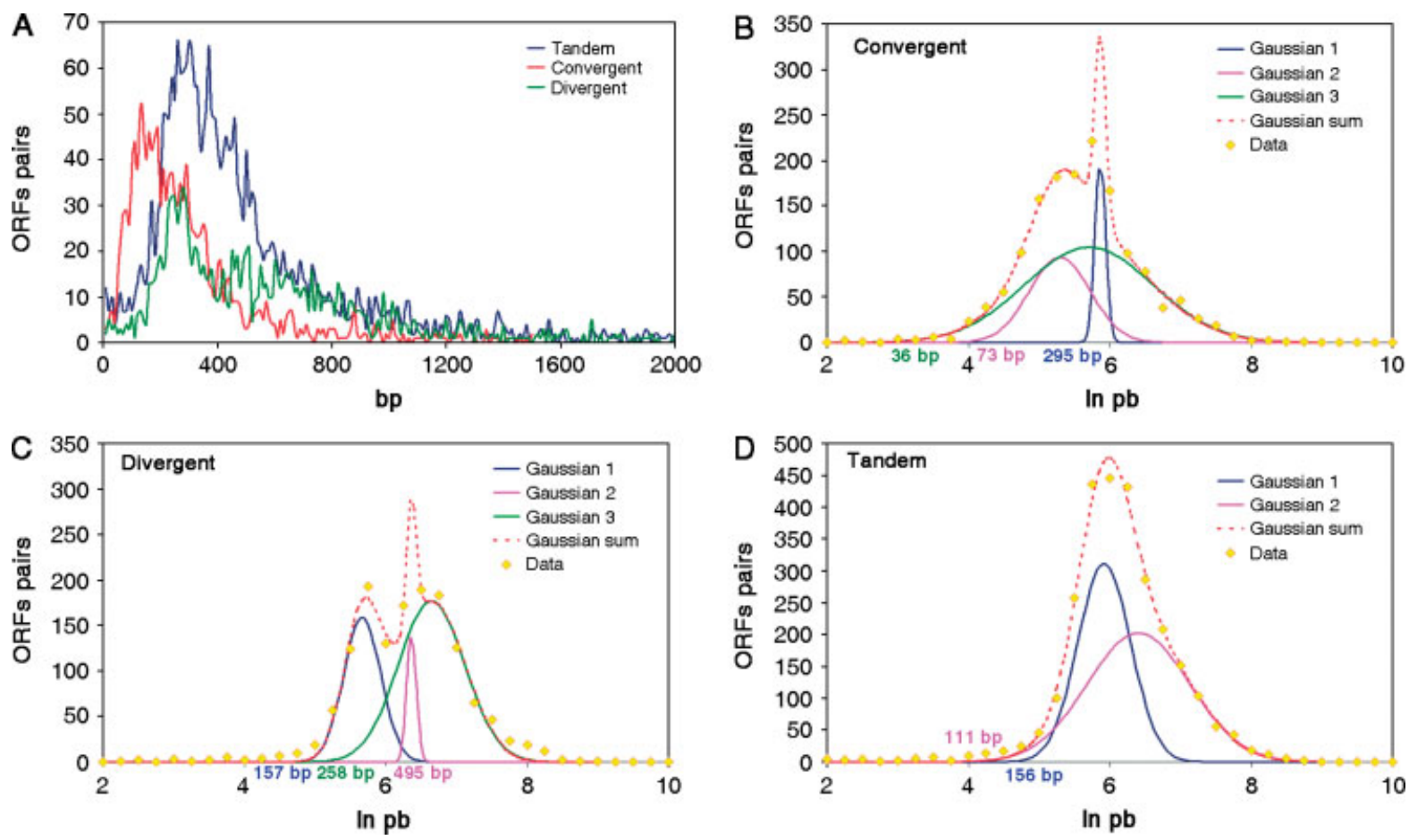

Figure I. Distances between ORFs in the yeast genome. Size distribution of separations between adjacent ORFs classified depending on the relative ORF orientation (A). Log normal curve deconvolution of the ORF distances for the three types of ORF orientation is shown in (B)-(D). A curve (dotted line) is fitted to the experimental data (yellow dots) using the non-linear least squares method. The curve is deconvoluted into two or three simpler Gaussians that represent ORFs subpopulations. The minimum separation for each subpopulation is calculated as the distance that leaves $5 \%$ of the population below it. These minimum distances are written in the same colour as the corresponding curve

terminator. The four possible combinations, using data from convergent and divergent ORFs, are 97, 116, 147 and $166 \mathrm{bp}$. This estimation is in agreement with the results obtained graphically for two subpopulations: 111 and $156 \mathrm{bp}$. These distances represent the average of the first two and the last two combinations, respectively.

The short distance observed for the group of genes that are closer to one another suggests that this class of genes may share regulatory elements and, therefore, may have a higher probability of being co-regulated. We have analysed the cosine values for co-regulation as described by Kemmeren et al. (2002). Figure 2 shows that all three classes of adjacent ORFs tend to be more co-regulated than the randomly generated pairs, as previously described by other authors (Cohen et al., 2000; Spellman and Rubin, 2002). A list of the most probable co-regulated divergent gene pairs is shown in Table 3 (a complete list is available from the authors). With regard to the two subgroups of divergent ORFs, we observed a significant increase in the frequency of co-regulation for the group with the shortest inter-ORF distance, as compared to either the other subgroup or any of the other groups analysed. This result is $99.99 \%$ significant using a $t$-test.

\section{In silico analysis of tandem ORFs}

If we assume that there is a minimum distance for adjacent tandem ORFs, the cases in which distances are shorter than the minimum should be either exceptions or erroneously annotated ORFs. We considered seven possible reasons for this:

1. There is a single gene, but due to sequencing errors it appears as two consecutive ORFs.

2. There is a single gene in strains other than S288c. In this background it would be a pseudogene containing two ORFs.

3. There is a single promoter and two genes generating a polycistronic mRNA. 

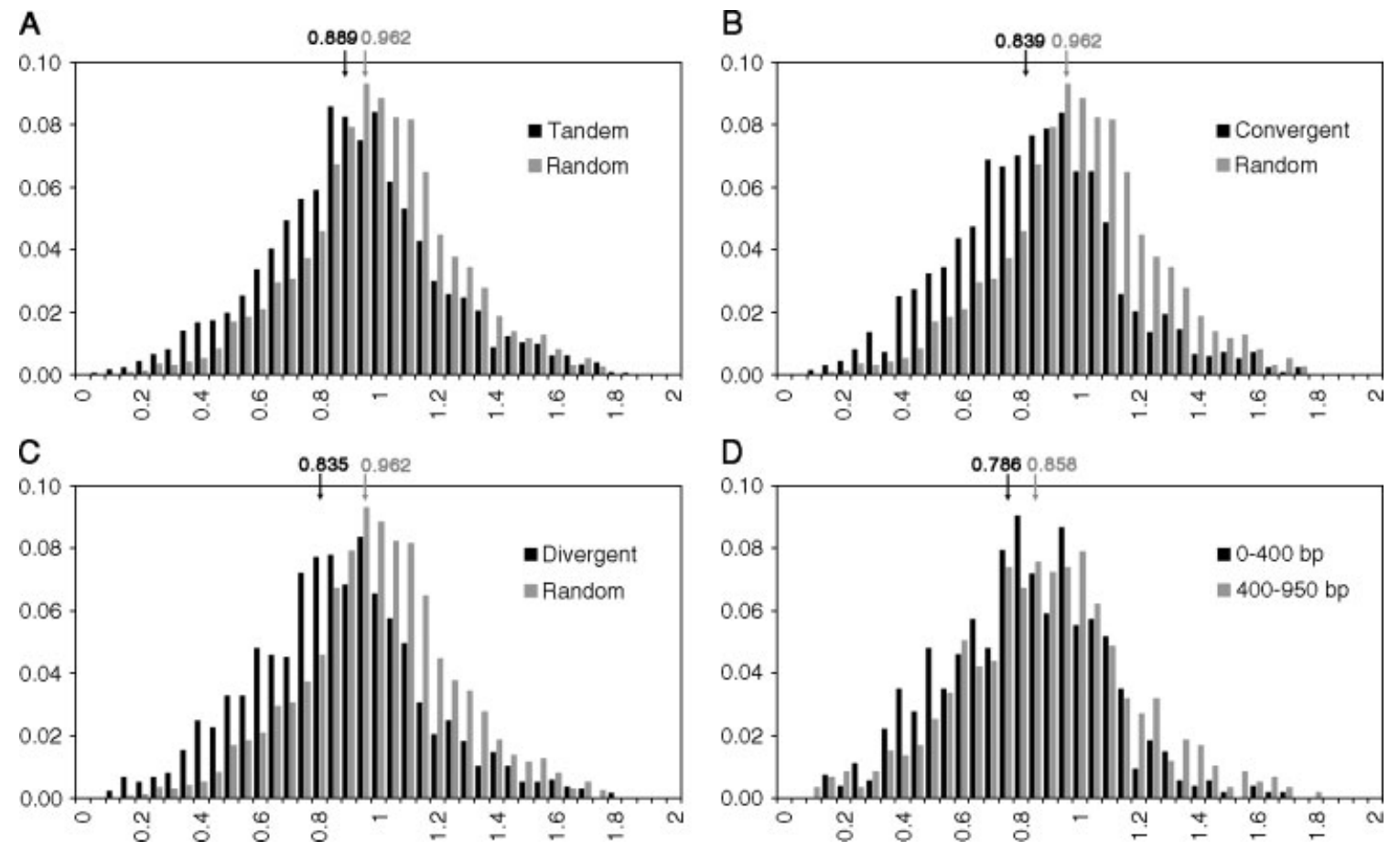

Figure 2. Histograms of the cosine co-regulation values for different adjacent ORF subpopulations. In $(A)-(C)$ the graphs show the analyses of the whole tandem, convergent or divergent ORF classes compared to the random pair distribution. A cosine value of 0 means perfect positive correlation, I means no correlation and 2 perfect negative correlation. In (D) the divergent ORFs were divided into two subgroups that correspond approximately to Gaussians I and 3 of Figure IC. The average value for cosine co-regulation is shown for every histogram. All the average pairs are $99.99 \%$ different using a $t$-test

4. One of the two ORFs is not a real gene.

5. There is an interrupting intron not annotated that, once spliced, converts them into a single ORF.

6. There is an annotation mistake that affects the ATG of the second ORF.

7. There is a naturally-programmed frameshift during ribosome scanning that generates a single protein.

For convergent and divergent ORFs, reasons 4 and $4 / 6$, respectively, can also apply. We decided to study tandem ORFs because they offer more possibilities.

Using information from the CYGD database (Güldener et al., 2005) in July 2002, when this study started, we found 159 ORFs pairs that were separated by 156 bp or less (Table 2). During the course of this study, new genome-wide data were published that compared the genome of S. cerevisiae with close relatives (Kellis et al., 2003; Cliften et al., 2003; Brachat et al., 2003; Dujon et al., 2004). These studies re-annotated the
ATG codons for many ORFs (possibility No. 6), discarded some spurious ORFs (possibility No. 4) and, finally, revealed some cases of pseudogenes (possibility No. 2). Moreover, the work of Namy et al. (2003) indicated that some tandem ORFs are part of a single protein because of a ribosome frame shift (possibility No. 7). After these corrections, many of the preselected pairs were no longer included in our list. Of the remaining 34 pairs, only 11 are below the shorter limit of $111 \mathrm{bp}$ described above. Our experimental study included six of the 34 pairs and 4 additional pairs that were selected before the reduction in the list (Table 2).

\section{Experimental testing of the different possibilities}

Before the publication of the genome sequence comparisons (Kellis et al., 2003; Cliften et al., 2003; Brachat et al., 2003; Dujon et al., 2004), we analysed the sequences of the ORF pairs $1-6$ by PCR amplification of the intergenic regions both in the S288c background and in a non-related strain 
Table 2. List of tandemly orientated ORF pairs separated by less than I56 bp

\begin{tabular}{|c|c|c|c|c|c|c|c|c|c|c|c|}
\hline \multicolumn{4}{|l|}{$\mathbf{p b}^{\$}$} & \multicolumn{4}{|l|}{ pb } & \multicolumn{4}{|l|}{ pb } \\
\hline 0 & & YDR504C & YDR505C & 65 & & YBL090W & YBL089W & 123 & & YKL137W & YKL136W \\
\hline 0 & & YIL168W & YIL167W & 66 & & YKL115C & YKL114C & 124 & & YGL169W & YGL168W \\
\hline 1 & & YGL165C & YGL164C & 67 & & YIL043C & YIL042C & 124 & & YMR068W & YMR069W \\
\hline 2 & & YIL171W & YIL170W & 67 & & YLR111W & YLR112W & 124 & & YPR095C & YPR096C \\
\hline 4 & & YBR026C & YBR027C & 68 & & YBR027C & YBR028C & $\begin{array}{l}125 \\
(299)\end{array}$ & 6 & YDR462W & YDR463W \\
\hline \multirow[t]{2}{*}{6} & & YBR226C & YBR227 & 73 & & YBL071C & YBL070C & 125 & & $\overline{\text { YJL007C }}$ & $\overline{\text { YJL006C }}$ \\
\hline & & & & & 1 & YPL271W & YPL270W & & 8 & YOR077W & YOR078W \\
\hline 6 & & YLR365W & YLR366W & 73 & & (ATP15) & (MDL2) & 127 & & (RTS2) & (BUD21) \\
\hline 7 & & YBL112C & YBL111C & 74 & & $\overline{\text { YMR084W }}$ & $\overline{\text { YMR085W }}$ & 128 & & $\overline{\text { YDR422C }}$ & $\overline{\text { YDR423C }}$ \\
\hline 7 & & YKL067W & YKL066W & 74 & & YPR168W & YPR169W & 129 & & YBR291C & YBR292C \\
\hline \multirow[t]{2}{*}{8} & & YLR101C & YLR102C & 76 & & YDR229W & YDR230W & 129 & & YDL016C & YDL015C \\
\hline & & & & & 4 & YJL089W & YJL088W & & & & \\
\hline 8 & & YML101C-A & YML101C & 77 & & $\begin{array}{l}\text { (SIP4) } \\
\end{array}$ & $\overline{(\text { ARG3) }}$ & 129 & & YGL212W & YGL211W \\
\hline 9 & & YIL165C & YIL164C & 78 & & $\overline{\text { YGL133W }}$ & $\overline{\text { YGL132W }}$ & 129 & & YGR201C & YGR202C \\
\hline \multirow[t]{2}{*}{11} & & YCR086W & YCR087W & 81 & & YDR431W & YDR432W & 129 & & YPR130C & YPR131C \\
\hline & & & & & & & & & 9 & & $\underline{\text { YJR121W }}$ \\
\hline 12 & & YDR024W & YDR025W & 81 & & YKL021C & YKL020C & 130 & & $\underline{\text { YJR120W }}$ & (ATP2) \\
\hline 12 & & YKL031W & YKL030W & 81 & & YOR300W & YOR301W & 131 & & $\overline{\text { YOR059C }}$ & $\overline{\text { YOR060C }}$ \\
\hline 12 & & YOL163W & YOL162W & 84 & & YML014W & YML013W & 132 & & YGL244W & YGL243W \\
\hline 13 & & YLR202C & YLR203C & 85 & & YLR373C & YLR374C & 132 & & YOR282W & YOR283W \\
\hline 16 & & YLR433C & YLR434C & 86 & & YBL063W & YBL062W & 133 & & YIR014W & YIR015W \\
\hline 16 & & YNL180C & YNL179C & 87 & & YNL320W & YNL319W & 134 & & YDR487C & YDR486C \\
\hline \multirow[t]{2}{*}{24} & & YDR157W & YDR158W & 89 & & YMR153C-A & YMR154C & 134 & & YGL053W & YGL052W \\
\hline & & & & 90 & 10 & YDR082W & YDR083W & & & & \\
\hline 24 & & YER119C & YER119C-A & (147) & & (STN1) & (RRP8) & 134 & & YKL208W & YKL207W \\
\hline 24 & & YJR023C & YJR024C & 91 & & $\overline{\text { YLR384C }}$ & $\overline{\text { YLR385C }}$ & 135 & & YCR013C & YCR014C \\
\hline 24 & & YLR311C & YLR312C & 92 & & YNL286W & YNL285W & 136 & & YDR396W & YDR395W \\
\hline 24 & & YNR065C & YNR066C & 95 & & YKL022C & YKL021C & 137 & & YDR203W & YDR204 \\
\hline 26 & & YIL025C & YIL024C & 95 & & YOL107W & YOL106W & 137 & & YDR400W & YDR401W \\
\hline 28 & & YDR290W & YDR291W & 95 & & YOR183W & YOR184W & 139 & & YKL111C & YKL110C \\
\hline 28 & & YIL086C & YIL085C & 96 & & YBR090C & YBR091C & 140 & & YNL046W & YNL045W \\
\hline 29 & & YEL034W & YEL033W & 99 & & YKR103W & YKR104W & 142 & & YMR075C-A & YMR076C \\
\hline 29 & & YFL057C & YFL056C & 101 & & YOR330C & YOR331C & 143 & & YCL059C & YCL058C \\
\hline 31 & & YLR393W & YLR394W* & 102 & & YAL046C & YAL045C & 143 & & YLR030W & YLR031W \\
\hline 32 & & YHL006C & YHL005C & 104 & & YGL129C & YGL128C & 143 & & YNL158W & YNL157W \\
\hline 33 & & YIL087C & YIL086C & 105 & & YKL044W & YKL043W & 146 & & YNL316C & YNL315C \\
\hline 39 & 2 & YHR130C & YHR131C** & 105 & & YML095C & YML095C-A & 146 & & YOL025W & YOL024W \\
\hline \multirow[t]{2}{*}{41} & & YGR044C & YGR045C & 105 & & YOL068C & YOL067C & 147 & & YCL009C & YCL008C \\
\hline & & & & & 3 & YMR063W & YMR064W & & & & \\
\hline 43 & & YGR025W & YGR026W & 106 & & (RIM9) & $\overline{\text { (AEP1) }}$ & 147 & & YDR219C & YDR220C \\
\hline 45 & & YOR014W & YOR015W & 107 & & $\overline{\text { YOR081C }}$ & $\overline{\text { YOR082C }}$ & 147 & & YGL046W & YGL045W \\
\hline 47 & & YEL046C & YEL045C & 107 & & YPR066W & YPR067W & 148 & & YER134C & YER135C \\
\hline 47 & & YGR163W & YGR164W & 110 & & YLR281C & YLR282C & 149 & & YML079W & YML078W \\
\hline 48 & & YOR024W & YOR025W & 111 & & YDR423C & YDR424C & 150 & & YLL031C & YLL030C \\
\hline 49 & & YPL278C & YPL277C & 111 & & YOL135C & YOL134C & 150 & & YNL149C & YNL148C \\
\hline 52 & & YER152C & YER153C & 112 & & YOR125C & YOR126C & 151 & & YGL231C & YGL230C \\
\hline & 7 & YNR056C & YNR057C & & & & & & & & \\
\hline (142) & & (BIO5) & $\overline{\text { (BIO4) }}$ & 114 & & YLR414C & YLR415C & 151 & & YGL035C & YGL034C \\
\hline 54 & & YBL049W & $\overline{\text { YBL048W }}$ & 114 & & YMR056C & YMR057C & 151 & & YHR110W & YHR111W \\
\hline 53 & & YGL241W & YGL240W & 115 & & YHL014C & YHL013C & 152 & & YMR151W & YMR152W \\
\hline 55 & & YGL128C & YGL127C & 115 & & YMR213W & YMR214W & 153 & & YAL045C & YAL044C \\
\hline 56 & & YJL097W & YJL096W & 116 & & YDL172C & YDL171C & 153 & & YDR204W & YDR205W \\
\hline 56 & & YJL021C & YJL020C & 116 & & YDR467C & YDR468C & 153 & & YHR057C & YHR058C \\
\hline \multirow[t]{2}{*}{56} & & YML032C & YML031C-A & 119 & & YFR046C & YFR047C & 154 & & YOR022C & YOR023C \\
\hline & & & & & 5 & YCL008C & YCL007C & & & & \\
\hline 56 & & YOR302W & YOR303W & 120 & & $\begin{array}{l}\text { (STP22) } \\
\end{array}$ & $\begin{array}{l}\text { CWH36) } \\
\end{array}$ & 154 & & YPL068C & YPL067C \\
\hline 57 & & YDL158C & YDL157C & 120 & & $\overline{\text { YDR023W }}$ & $\overline{\text { YDR024W }}$ & 155 & & YGL005C & YGL004C \\
\hline 57 & & YER039C & YER039C-A & 121 & & YMR294W-A & YMR294W & 155 & & YGR164W & YGR165W \\
\hline 61 & & YKL084W & YKL083W & 122 & & YBR032W & YBR033W & 155 & & YMR103C & YMR104C \\
\hline 63 & & YKR072C & YKR073C & 122 & & YCR085W & YCR086W & 156 & & YNL129W & YNL128W \\
\hline
\end{tabular}

* The 34 pairs that are separated by less than 156 bp after sequence and ATG correction updates are in bold.

** The experimentally studied cases are highlighted and numbered.

\$ The distances shown are those initially assigned in the CYGD data bank (July 2002). In some cases, the newly assigned distance is shown in brackets. 
Table 3. List of the 75 divergently orientated ORF pairs separated by less than 400 bp that are likely to share regulatory elements

\begin{tabular}{|c|c|c|c|c|c|c|c|c|}
\hline \multicolumn{2}{|c|}{ Divergent ORFs* } & \multirow{2}{*}{$\begin{array}{c}\begin{array}{c}\text { Cosine } \\
\text { correlation }\end{array} \\
0.104\end{array}$} & \multicolumn{2}{|c|}{ Divergent ORFs } & \multirow{2}{*}{$\begin{array}{c}\begin{array}{c}\text { Cosine } \\
\text { correlation }\end{array} \\
0.356\end{array}$} & \multicolumn{2}{|c|}{ Divergent ORFs } & \multirow{2}{*}{$\begin{array}{c}\begin{array}{c}\text { Cosine } \\
\text { correlation }\end{array} \\
0.431\end{array}$} \\
\hline YNL062C & YNL06IW & & YLR065C & YLR066W & & YHRI07C & YHRI08W & \\
\hline YHR065C & YHR066W & 0.104 & YPL246C & YPL245W & 0.359 & YLR203C & YLR204W & 0.432 \\
\hline YERI26C & YERI27W & 0.127 & YFR04IC & YFR042W & 0.362 & YPR085C & YPR086W & 0.443 \\
\hline YNL248C & YNL247W & 0.129 & YLL036C & YLL035W & 0.369 & YNL334C & YNL333W & 0.450 \\
\hline YPL2I2C & YPL2IIW & 0.155 & YFL060C & YFL059W & 0.370 & YPL094C & YPL093W & 0.450 \\
\hline YAL036C & YAL035W & 0.220 & YML047C & YML046W & 0.371 & YILI54C & YILI53W & 0.451 \\
\hline YKLI44C & YKLI43W & 0.227 & YHR069C & YHR070W & 0.371 & YKLI74C & YKLI73W & 0.453 \\
\hline YERI42C & YERI43W & 0.237 & YORI64C & YORI65W & 0.375 & YDL226C & YDL225W & 0.457 \\
\hline YORI67C & YORI68W & 0.238 & YLR209C & YLR2IOW & 0.375 & YDR067C & YDR068W & 0.458 \\
\hline YNL002C & YNL00IW & 0.247 & YFR003C & YFR004W & 0.376 & YDR329C & YDR330W & 0.461 \\
\hline YKR024C & YKR025W & 0.264 & YIL098C & YIL097W & 0.392 & YGRI72C & YGRI73W & 0.462 \\
\hline YBRI4IC & YBRI42W & 0.288 & YEROI8C & YEROI9W & 0.392 & YGR078C & YGR079W & 0.462 \\
\hline YIL020C & YILOI9W & 0.293 & YKR043C & YKR044W & 0.393 & YMRI59C & YMRI60W & 0.466 \\
\hline YFL002C & YFLOOIW & 0.310 & YBLOIOC & YBL009W & 0.397 & YCL033C & YCL032W & 0.467 \\
\hline YDRI20C & YDRI2IW & 0.310 & YPL004C & YPL003W & 0.398 & YDRI96C & YDR197W & 0.469 \\
\hline YILI04C & YILI03W & 0.314 & YGL003C & YGL002W & 0.398 & YNL074C & YNL073W & 0.470 \\
\hline YLL062C & YLL06IW & 0.325 & YGL048C & YGL047W & 0.401 & YHR024C & YHR025W & 0.472 \\
\hline YAL065C & YAL064W-B & 0.326 & YML023C & YML022W & 0.401 & YBR264C & YBR265W & 0.477 \\
\hline YPRI86C & YPRI87W & 0.330 & YOR219C & YOR220W & 0.406 & YLROI4C & YLROI5W & 0.487 \\
\hline YNL294C & YNL293W & 0.334 & YIR036C & YIR037W & 0.408 & YCR002C & YCR003W & 0.488 \\
\hline YKR08IC & YKR082W & 0.335 & YKL074C & YKL073W & 0.412 & YBR245C & YBR246W & 0.491 \\
\hline YJR073C & YJR074W & 0.337 & YJR049C & YJR050W & 0.415 & YOR288C & YOR289W & 0.491 \\
\hline YGL246C & YGL245W & 0.344 & YJLI92C & YJL191W & 0.416 & YOR035C & YOR036W & 0.492 \\
\hline YBR046C & YBR047W & 0.351 & YBL057C & YBL056W & 0.420 & YPL20IC & YPL200W & 0.494 \\
\hline YDR020C & YDR02IW & 0.354 & YOL077C & YOL076W & 0.421 & YML06IC & YML060W & 0.498 \\
\hline
\end{tabular}

* Only those ORF pairs that do not include a dubious one (according to SGD).

** Defined in Materials and methods. Note that the lower the value of cosine, the higher the correlation.

(T73). In all cases, we detected no differences in DNA sequence that could convert the ORF pair into a single ORF (not shown). This discarded possibilities Nos. 1 and 2 for these ORF pairs.

We performed RT-PCR on total RNA from the S288c strain using the same oligonucleotides (priming inside the two consecutive ORFs) to check for the existence of an intron. In all cases, we obtained a fragment with the same size as that obtained from genomic DNA (see Figure 3B for two examples). This result allowed us to discard possibility No. 5 , but demonstrates that an mRNA extends over the region of the two ORFs, supporting possibility No. 3 .

In order to check for the existence of polycistronic mRNAs in the 10 ORF pairs studied, we used the $3^{\prime}$ RACE method to map the ends of the mRNAs. Figure $3 \mathrm{~A}$ and Table 4 show the $3^{\prime}$ ends of the mRNAs. As shown, we observed that in most cases mRNAs have alternative $3^{\prime}$ ends. In two cases, ORF pairs 7 and 10, the mRNA does not
Table 4. List of mRNA overlaps between experimentally studied tandemly orientated ORF pairs

Number of nucleotides that the mRNA extends

Pair** beyond the stop codon of the first gene of the pair

\begin{tabular}{|c|c|}
\hline I & Approximately 260 and $210 \mathrm{nt}$ \\
\hline 2 & Approximately 180 and 280 nt \\
\hline 3 & $\begin{array}{l}988,1109,1789 \text { and } 1879 \mathrm{nt}^{*} \text { (the last two mRNA } \\
\text { completely cover both ORFs) }\end{array}$ \\
\hline 4 & Approximately 90, 140, $410 \mathrm{nt}$ \\
\hline 5 & Could not be determined in this study \\
\hline 6 & $\begin{array}{l}\text { A population of mRNA that extends approximately } \\
\text { between } 160 \text { and } 210 \mathrm{nt}\end{array}$ \\
\hline 7 & 89 and $113 \mathrm{nt}^{*}$ \\
\hline 8 & $841 \mathrm{nt}^{*}$ (completely covers both ORFs) \\
\hline 9 & Could not be determined in this study \\
\hline ๑ & I 47 nt* (match exactly with the second ORF start) \\
\hline
\end{tabular}

* Confirmed by sequencing.

** Numbers as in Table 2.

enter the next ORF, although it should overlap with the $5^{\prime}$ end of the next ORF mRNA. In the rest of the cases, however, the mRNA enters extensively 


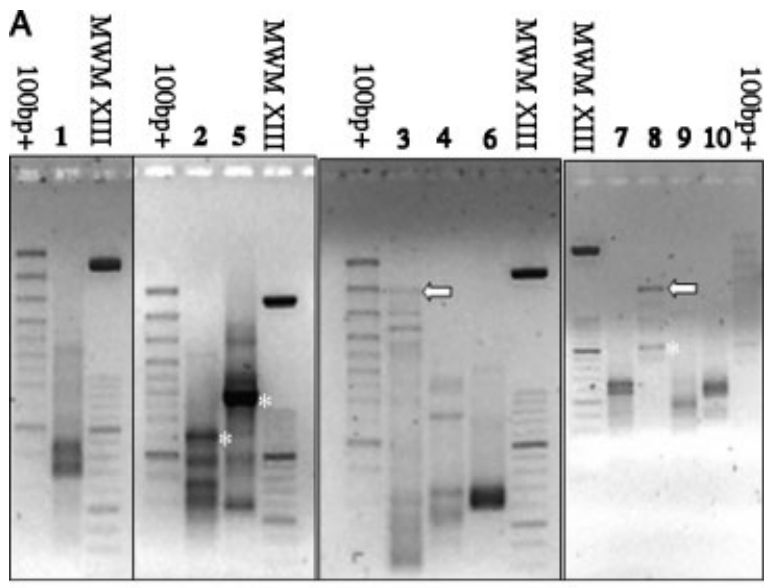

B

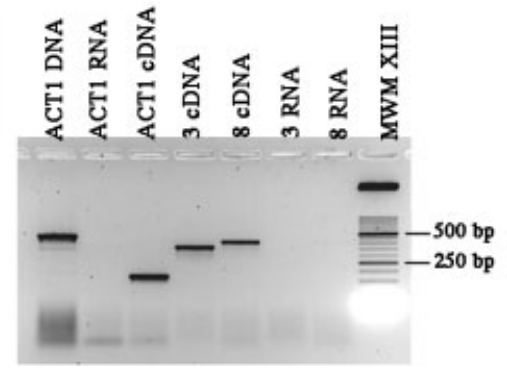

Figure 3. Identification of polycistronic mRNAs. (A) $3^{\prime}$ RACE of the mRNAs of several ORF pairs. The agarose gel electrophoresis shows the result of RACE bands of the candidates, numbered as in Table 4. The white arrows mark the sequenced fragments that correspond to mRNAs completely covering the adjacent next ORF. The asterisks indicate unspecific bands discarded after sequencing. Molecular weight markers used were MWM XIII from Roche (50 bp ladder from 50 to 750 bp) and Gene Ruler $100 \mathrm{bp}+$ ladder from MBI Fermentas. (B) RT-PCR analysis of intergenic region between ORF pairs 3 and 8 . In both loci, a band (obtained by RT-PCR from oligonucleotides marked in Figure 4B) that spans both ORFs is seen. ACTI is used as a control because it contains an intron. DNA contamination in the RNA sample would give a 480 bp band (lane ACTIDNA). This band is not present in the RNA sample either before (ACTIRNA) or after RT (ACTICDNA). A shorter band of 172 bp due to intron splicing is seen instead in the cDNA sample, which demonstrates that the bands of $367 \mathrm{bp}$ and $418 \mathrm{bp}$ seen in samples $3 c$ DNA and $8 c$ DNA are not due to DNA contamination

into the next ORF. In two cases, ORF pairs 3 and 8 , the sizes of the mRNAs detected (Figure 3A) and the RT-PCR amplification of the intergenic region (Figure 3B) suggests the existence of complete polycistronic mRNAs. In order to precisely map the $3^{\prime}$ ends of these mRNAs, we sequenced the RACE bands for pairs 3 and 8 . The analysis of pair 3
A
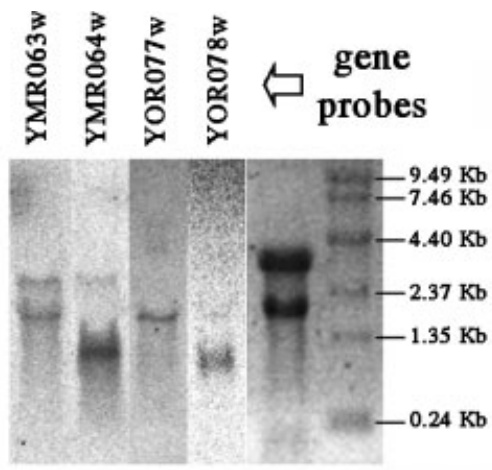

B
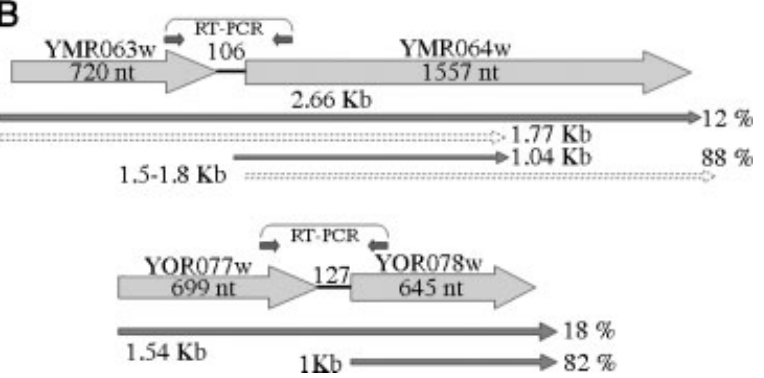

Figure 4. Transcription scheme of two selected loci. (A) Northern blot for confirmation of the mRNA sizes of the ORF pairs 3 and 8. Lanes 5, 6 on the right show ethidium bromide stain of the sample and an RNA size marker. Lanes I-4 show the hybridization of lane 5 successively with the gene probes. (B) Model for the transcripts encoded by the two ORF pairs analysed. The regions covered by the different mRNAs are depicted. Results were obtained from the $3^{\prime}$ RACE (Figure 3A) and Northern analyses. The oligonucleotides used for RT-PCR (Figure $3 B$ ) are shown. The left one was also used for $3^{\prime}$ RACE (Figure 3A). A previously described I.5- I.8 kb mRNA (Payne et al., 1993) that we did not detect and a $1.77 \mathrm{~kb}$ mRNA only detected with YMR063w probe are shown as a dotted line. The relative amount of each mRNA is marked

indicated that an mRNA from YMR063W (RIM9) enters YMR064W (AEP1) to positions 1708, 1829, 2509 and $2599 \mathrm{bp}$ from the ATG of the RIM9. The longest mRNA completely covers the AEP1 ORF (see Figure 4B). For ORF pair 8, there is an mRNA which extends to position 1540 from the ATG that also covers the second ORF, YOR078W (BUD21) (see Figure 4B). In order to further confirm the RACE results, to evaluate the relative abundance of the mRNA species and to position the $5^{\prime}$ ends of the mRNAs, we performed a series of Northern blots and hybridizations with probes from the two ORFs from each pair (Figure 4A). The interpretation of the results from the $3^{\prime}$ RACE and Northern analysis is shown in Figure 4B. Our results 
on YMR064W coincide in part with those published by Payne et al. (1993), who found several mRNAs, including those of 2.66 and $1.77 \mathrm{~kb}$. With regard to the YOR077-078W pair, our results are compatible with those of Hurowitz et al. (2003), who found, apart from the expected $1 \mathrm{~kb}$ mRNA for $B U D 21$, a minor long mRNA that they considered a false positive. We did not detect a short mRNA for YOR077W (RTS2) in our experimental conditions.

\section{Discussion}

The representation of inter-ORF distances in yeast appears to correspond to a log-Normal distribution. A simple explanation for such a distribution is that it is caused by a limit in size in the left part (the minimum distance allowed) and a random distribution on the other side (there is no maximum distance in between ORFs). The deconvolution of the curves into simpler Gaussians allows us to determine that all three populations seem to be composed of at least two or three groups of inter ORF distances.

Using the distributions, we can calculate the typical and the minimum size for each of the groups. Using the reasoning of Dujon (1996), the distance in between two divergent ORFs corresponds to the sizes of two adjacent promoters and the distance in between two convergent ORFs corresponds to the sizes of two adjacent terminators. The sizes calculated, thus, for the two main groups of gene promoters would be 145 and 386 bp with minima of 79 and $129 \mathrm{bp}$, respectively. What could be the reason for the existence of at least two different populations of gene promoters? One plausible explanation is the allowance of a partial overlap between promoters. The smaller size group would correspond to overlapping promoters in which some non-essential elements could be interspersed, or even shared between the two genes. If this were true, the genes belonging to this group would have a higher probability of being co-regulated. Our analysis (Figure 2) demonstrates that, in fact, this is the case. One can imagine that a gene promoter should have essential elements, such as TATA and Inr, which cannot be shared because of their unidirectionality. These elements tend to be very close to the ORF's ATG. Other non-essential elements, however, can be either bidirectional or, because of their non-essential role, can be placed alternatively within the region in between two divergent ORFs. The first option would give rise to co-regulated genes and would explain our results. Several examples of co-regulated divergent yeast genes are already known (e.g. GAL1-GAL10, or the cases listed in Wade et al., 2006). Although this hypothesis could not be proved in a previous analysis made by Cohen et al. (2000), we think that our approach, which differentiates between subgroups of divergent genes, strongly supports the hypothesis that one of the main reasons for co-regulation is the use of partially shared promoters. We postulate that many others will be discovered in the future and that the probability of such cases will be higher for genes belonging to the closest distance subgroup. A list of the most likely cases is shown in Table 3 . Similar arguments can be used for terminators but, in that case, co-regulation will not be a biological consequence of overlapping. For tandem genes, combinations of overlapping and non-overlapping promoters and terminators can exist as well.

The hypothesis of the existence of a minimum distance necessary for the separation of nonoverlapping elements raises the question of why there are some exceptions for the three cases. Are they real exceptions or do they correspond to erroneously annotated ORFs? We selected the case of tandem ORFs to experimentally address these questions. The fact that $79 \%$ of the 159 initially selected ORFs pairs in July 2002 were discarded by several studies (Kellis et al., 2003; Cliften et al., 2003; Brachat et al., 2003; Dujon et al., 2004; Namy et al., 2003) suggests that, for the vast majority of cases, annotation errors are the cause for the short distance. Our results on a sample of the rest of the cases also support this hypothesis (see below).

Our experimental study on a sample of the tandem ORF pairs concludes that, in most cases, the close proximity between two ORFs leads to invasion of the upstream mRNA into the region covered by the downstream ORF. This situation may be not neutral for the second gene. It has been demonstrated previously that TI can occur when an RNA polymerase enters the promoter of the downstream gene. TI can occur when mutations occur in the terminator of the first gene (Greger and Proudfoot, 1998; Valerius et al., 2002). However, it is more interesting to note that there are cases in 
which wild-type sequences allow TI on the downstream gene (Greger and Proudfoot, 1998; Greger et al., 2000; Martens et al., 2004). In these cases, RNA polymerases invade the promoter, causing promoter occlusion by affecting transcription factor binding (Greger et al., 2000; Martens et al., 2004) and they can even transcribe the downstream ORF, producing bicistronic mRNAs (Gerger and Proudfoot, 1998). This has been shown for tandem ORFs that are separated by 191 bp (FBP1-PSY3), 417 bp (ARO4-HIS7) or 600 bp (GAL10-GAL7) (Springer et al., 1997; Aranda et al., 1998b; Greger et al., 2000). In some cases, however, no large effects on downstream transcription by TI were observed (Aranda et al., 1998b). Because changes in efficiency of both the promoter and terminator affects TI (Springer et al., 1997; Greger et al., 1998), it seems likely that a fine tuning of the $3^{\prime}$ end formation signal to the promoter strength of the upstream mRNA is necessary to prevent TI from the adjacent gene.

In conclusion, given that even tandem ORFs separated by $600 \mathrm{bp}$ are susceptible to TI, it is conceivable that most of the members of this group of ORFs are potentially affected by TI (74\% of the tandem ORFs are under this distance). The cell has developed mechanisms to avoid such perturbations, such as efficient terminators, DNA-binding factors or/and nucleosomal organization (Valerius et al., 2002). Whatever the mechanisms, the shorter the distance between ORFs, the more difficult their use. This may be one of the reasons for the existence of a minimum separation distance. Another reason may be that, although the promoter and the terminator of two successive genes can be interspersed (Aranda et al., 1998b; Springer et al., 1997), this is again more difficult for shorter distances. We believe that in some cases (as in pairs 3 and 8 shown in this study) TI is not efficiently avoided and this results in minor polycistronic mRNAs. Whether or not these mRNAs are functional, especially for the translation of the second ORF, is a question for further investigation.

\section{Acknowledgements}

This work was supported by Grants GEN2001-4707-C0807 and BMC2003-07072-C03-02 from the Ministerio de Educación y Ciencia (to J.E.P-O) and Grupos 03/096 from the Generalitat Valènciana to Vicente Tordera. V.P. is a fellowship holder of the Consellería de Educació i Ciència de la Generalitat Valènciana.

\section{References}

Aranda A, Pérez-Ortín JE, Moore C, del Olmo M. 1998a. Transcription termination downstream of the Saccharomyces cerevisiae $F B P 1$ poly(A) site does not depend on efficient $3^{\prime}$ end processing. RNA 4: 303-318.

Aranda A, Pérez-Ortín JE, Moore C, del Olmo M. 1998b. The yeast FBP1 poly(A) signal functions in both orientations and overlaps with a gene promoter. Nucleic Acids Res 26: 4588-4596.

Atkins D, Arndt GM, Izant JG. 1994. Antisense gene expression in yeast. Biol Chem Hoppe-Seyler 375: 721-729.

Brachat S, Dietrich FS, Voegeli S, et al. 2003. Reinvestigation of the Saccharomyces cerevisiae genome annotation by comparison to the genome of a related fungus: Ashbya gossypii. Genome Biol 4: R45.

Cliften P, Sudarsanam P, Desikan A, et al. 2003. Finding functional features in Saccharomyces genomes by phylogenetic footprinting. Science 301: 71-76.

Cohen BA, Mitra RD, Hughes JD, Church GM. 2000. A computational analysis of whole-genome expression data reveals chromosomal domains of gene expression. Nat Genet 26: $183-186$.

Dujon B. 1996. The yeast genome project: what did we learn? Trends Genet 12: 263-270.

Dujon B, Sherman D, Fischer G, et al. 2004. Genome evolution in yeasts. Nature 430: $35-44$.

Frohman MA, Dush MK, Martin GR. 1988. Rapid production of full-length cDNAs from rare transcripts: amplification using a single gene-specific oligonucleotide primer. Proc Natl Acad Sci USA 85: 8998-9002.

Eggermont J, Proudfoot NJ. 1993. Poly(A) signals and transcriptional pause sites combine to prevent interference between RNA polymerase II promoters. EMBO J 12: 2539-2548.

García-Martínez J, Aranda A, Pérez-Ortín JE. 2004. Genomic Run-On evaluates transcription rates for all yeast genes and identifies gene regulatory mechanisms. Mol Cell 15: 303-313.

Greger IH, Aranda A, Proudfoot N. 2000. Balancing transcriptional interference and initiation on the GAL7 promoter of Saccharomyces cerevisiae. Proc Natl Acad Sci USA 97: 8415-8420.

Greger IH, Proudfoot NJ. 1998. Poly(A) signals control both transcriptional termination and initiation between the tandem GAL10 and GAL7 genes of Saccharomyces cerevisiae. EMBO J 16: 4771-4779.

Güldener U, Münsterkotter M, Kastenmuller G, et al. 2005. CYGD: the Comprehensive Yeast Genome Database. Nucleic Acids Res 33: D364-D368.

van Helden J, del Olmo M, Pérez-Ortín JE. 2000. Statistical analysis of yeast genomic downstream sequences reveals putative polyadenylation signals. Nucleic Acids Res 28: 1000-1010.

Hoffman CS, Winston F. 1987. A ten-minute DNA preparation from yeast efficiently releases autonomous plasmids for transformation of Escherichia coli. Gene 57: 267-272.

Hurowitz EH, Brown PO. 2003. Genome-wide analysis of mRNA lengths in Saccharomyces cerevisiae. Genome Biol 5: R2.

Kellis M, Patterson N, Endrizzi M, Birren B, Lander ES. 2003. Sequencing and comparison of yeast species to identify genes and regulatory elements. Nature 423: 241-254. 
Kemmeren P, van Berkum NL, Vilo J, et al. 2002. Protein interaction verification and functional annotation by integrated analysis of genome-scale data. Mol Cell 9: 1133-1143.

Marín A, Wang M, Gutiérrez G. 2004. Short-range compositional correlation in the yeast genome depends on transcriptional orientation. Gene 333: 151-155.

Martens JA, Laprade L, Winston F. 2004. Intergenic transcription is required to repress the Saccharomyces cerevisiae SER3 gene. Nature 429: 571-574.

Namy O, Duchateau-Nguyen G, Hatin I, et al. 2003. Identification of stop codon readthrough genes in Saccharomyces cerevisiae. Nucleic Acids Res 9: 2289-2296.

Prescott EM, Proudfoot NJ. 2002. Transcriptional collision between convergent genes in budding yeast. Proc Natl Acad Sci USA 99: 8796-8801.

Puig S, Pérez-Ortín JE, Matallana E. 1999. Transcriptional and structural study of a region of two convergent overlapping yeast genes. Curr Microbiol 39: 369-373.
Querol A, Ramón D. 1996. The application of molecular techniques in wine microbiology. Trends Food Sci Technol 7: 73-78.

Spellman PT, Rubin GM. 2002. Evidence for large domains of similarly expressed genes in the Drosophila genome. J Biol 1: 5 .

Springer C, Valerius O, Strittmatter A, Braus GH. 1997. The adjacent yeast genes ARO4 and HIS7 carry no intergenic region. J Biol Chem 272: 26318-26324.

Valerius O, Brendel C, Duvel K, Braus GH. 2002. Multiple factors prevent transcriptional interference at the yeast ARO4-HIS7 locus. J Biol Chem 24: 21440-21445.

Wade C, Umbarger M, McAlear M. 2006. The budding yeast rRNA and ribosome biosynthesis (RRB) regulon contains over 200 genes. Yeast 23: 293-306.

Zhang Z, Dietrich FS. 2005. Mapping of transcription start sites in Saccharomyces cerevisiae using 5' SAGE. Nucleic Acids Res 33: $2838-2851$. 